\title{
An open-label, multicenter, phase lb study investigating the effect of apalutamide on ventricular repolarization in men with castration- resistant prostate cancer
}

\author{
Bodine P. S. I. Belderbos ${ }^{1} \cdot$ Ronald de Wit ${ }^{1} \cdot$ Caly Chien $^{2} \cdot$ Anna Mitselos $^{3} \cdot$ Peter Hellemans ${ }^{3} \cdot$ James Jiao $^{4}$. \\ Margaret K. $\mathrm{Yu}^{5} \cdot$ Gerhardt Attard $^{6} \cdot$ lurie Bulat $^{7} \cdot$ W. Jeffrey Edenfield ${ }^{8} \cdot$ Fred Saad $^{9}$
}

Received: 28 March 2018 / Accepted: 22 June 2018 / Published online: 5 July 2018

(c) The Author(s) 2018

\begin{abstract}
Purpose Phase Ib study evaluating the effect of apalutamide, at therapeutic exposure, on ventricular repolarization by applying time-matched pharmacokinetics and electrocardiography (ECG) in patients with castration-resistant prostate cancer. Safety of daily apalutamide was also assessed.

Methods Patients received $240 \mathrm{mg}$ oral apalutamide daily. Time-matched ECGs were collected via continuous 12-lead Holter recording before apalutamide (Day - 1) and on Days 1 and 57 (Cycle 3 Day 1). Pharmacokinetics of apalutamide were assessed on Days 1 and 57 at matched time points of ECG collection. QT interval was corrected for heart rate using Fridericia correction $(\mathrm{QTcF})$. The primary endpoint was the maximum mean change in $\mathrm{QTcF}(\triangle \mathrm{QTcF})$ from baseline to Cycle 3 Day 1 (steady state). Secondary endpoints were the effect of apalutamide on other ECG parameters, pharmacokinetics of apalutamide and its active metabolite, relationship between plasma concentrations of apalutamide and QTcF, and safety. Results Forty-five men were enrolled; $82 \%$ received treatment for $\geq 3$ months. At steady state, the maximum $\Delta \mathrm{QTcF}$ was $12.4 \mathrm{~ms}$ and the upper bound of its associated $90 \%$ CI was $16.0 \mathrm{~ms}$. No clinically meaningful effects of apalutamide were reported for heart rate or other ECG parameters. A concentration-dependent increase in QTcF was observed for apalutamide. Most adverse events (AEs) (73\%) were grade 1-2 in severity. No patients discontinued due to QTc prolongation or AEs. Conclusion The effect of apalutamide on QTc prolongation was modest and does not produce a clinically meaningful effect on ventricular repolarization. The AE profile was consistent with other studies of apalutamide.
\end{abstract}

Keywords Apalutamide · Castration-resistant prostate cancer · Ventricular repolarization · QT interval · Pharmacokinetics

Ronald de Wit

r.dewit@erasmusmc.nl

1 Erasmus MC Cancer Institute, Dr. Molewaterplein 40, 3015 GD Rotterdam, The Netherlands

2 Janssen Research \& Development, 1400 McKean Road, Spring House, PA 19477, USA

3 Janssen Research \& Development BE, Turnhoutseweg 30, Beerse, Belgium

4 Janssen Research \& Development, 920 Route 202 South, Raritan, NJ 08869, USA

5 Janssen Research \& Development, 10990 Wilshire Blvd., Suite 1200, Los Angeles, CA 90024, USA
6 Research Department of Oncology, UCL Cancer Institute, 72 Huntley Street, London WC1E 6DD, UK

7 ARENSIA Exploratory Medicine's Research Unit, The Institute of Oncology, 30 N.Testemitanu str., 2025 Chişinău, Republic of Moldova

8 GHS Cancer Institute, 900 West Faris Road, Greenville, SC 29615, USA

9 Centre Hospitalier de l'Université de Montréal/CRCHUM, 900, rue St-Denis, porte R04-446, Montreal, Québec H2X 0A9, Canada 


\section{Introduction}

Prostate cancer is the second most common cancer in men worldwide, accounting for $15 \%$ of cancers diagnosed in men [1]. Metastatic castration-resistant prostate cancer (mCRPC) is associated with progressive morbidities, including skeletal-related events [2]. Because prostate cancer cells depend on the androgen receptor (AR) for survival and growth, treatment for recurrent or primary metastatic prostate cancer targets this receptor axis [3]. Despite initial therapies that target the AR, many patients progress to CRPC [3]. Apalutamide is an orally administered next-generation AR inhibitor currently approved in the United States for patients with nonmetastatic CRPC (nmCRPC) [4]. It directly binds the ligand-binding domain of the AR, inhibits AR nuclear translocation and DNA binding, and impedes AR-mediated transcription [5].

The efficacy and safety of apalutamide were demonstrated in patients with prostate cancer in the SPARTAN study, a randomized, double-blind, placebo-controlled, multicenter trial that evaluated apalutamide treatment in 1207 patients with high-risk nmCRPC [6]. This study was the first to demonstrate a significantly longer median metastasis-free survival (MFS; 2 years over placebo) in apalutamide-treated patients compared with placebotreated patients, with consistent benefit for apalutamide across all secondary endpoints, including time to symptomatic progression [6]. Minimal cardiac adverse events (AEs) were observed; atrial fibrillation was cited as the primary cardiac-associated AE reason for dose interruption and occurred in 0.7 and $0.5 \%$ of patients in the apalutamide and placebo arms, respectively. Based on these data, apalutamide was approved in February 2018 by the US Food and Drug Administration (FDA) for the treatment of men with nmCRPC [4].

Apalutamide pharmacokinetics (PK) has been well characterized in clinical studies. Apalutamide is rapidly absorbed, with a median time to maximum observed plasma concentration $\left(C_{\max }\right)$ of $2-3 \mathrm{~h}$ after oral administration [7]. Additionally, PK was approximately proportional across dose levels, with a mean effective half-life of approximately 3 days after multiple doses (Data on file, Janssen). Steady state exposure was achieved following 4 weeks of continuous, daily apalutamide administration $[4,7]$. For $N$-desmethyl apalutamide, a minimal peak to trough fluctuation ratio in plasma at steady state $(\approx 1.3)$ was observed. Time to reach $C_{\max }\left(t_{\max }\right)$ for $N$-desmethyl apalutamide at steady state was variable and typically occurred at around 1 or $24 \mathrm{~h}$ post dose (Data on file, Janssen).

The preclinical cardiovascular safety of apalutamide and its active metabolite $N$-desmethyl apalutamide has been assessed in in vitro and in vivo studies (Data on file, Janssen). Both apalutamide and $N$-desmethyl apalutamide inhibited human ether-à-go-go-related (hERG) gene current, with half maximal inhibitory concentration $\left(\mathrm{IC}_{50}\right)$ values of 6.17 and $4.56 \mu \mathrm{M}$, respectively, representing a safety margin of at least seven relative to the anticipated $C_{\max }$ for unbound apalutamide and $N$-desmethyl apalutamide in patients at the clinical dose of $240 \mathrm{mg} /$ day (Data on file, Janssen). No relevant effect was produced (no prolongation in action potential duration; no effect on resting membrane potential) in isolated canine Purkinje fibers with up to $30 \mu \mathrm{M}$ of apalutamide or $N$-desmethyl apalutamide (Data on file, Janssen). Preclinical in vitro receptor binding assay testing did not reveal an effect of apalutamide on $\mathrm{Na}^{+}$or $\mathrm{Ca}^{2+}$ channels (Data on file, Janssen). No in vivo treatment-related cardiovascular effects (blood pressure, heart rate, body temperature, ECG lead II intervals, PR, QRS, QT, QTc, or ECG waveform morphology) were noted in a single-dose telemetry study in conscious dogs up to $40 \mathrm{mg} / \mathrm{kg}$ or after repeated dosing in good laboratory practice toxicology studies in dogs up to $10 \mathrm{mg} / \mathrm{kg} /$ day with exposures in the range of the clinical exposure for apalutamide and its metabolite $N$-desmethyl apalutamide (Data on file, Janssen). Overall preclinical cardiovascular safety assessment of apalutamide was not indicative of an increased risk for QTc prolongation in clinical use (Data on file, Janssen).

The effect of apalutamide on ventricular repolarization was previously evaluated as part of a phase I/II study [7] that included time-matched triplicate 12-lead electrocardiograms (ECGs) collected at baseline and at steady state in 12 patients with CRPC. The data showed no significant effect from apalutamide on ECG parameters, and there was no conclusive evidence for an increase in Fridericia corrected QT interval (QTcF) (Data on file, Janssen).

Androgen deprivation therapy (ADT) can increase cardiovascular risk because of its adverse effect on risk factors for cardiovascular disease [8, 9]. Combination treatment with bicalutamide plus LHRH agonist therapy and 150-mg bicalutamide monotherapy may lead to QTc prolongation [10-12]. In AFFIRM, a randomized phase III study, the effect of enzalutamide ( $160 \mathrm{mg} /$ day) on QTcF was assessed at steady state in 800 patients with CRPC [13]. No clinically meaningful changes were observed between the mean QTcF interval change from baseline in patients treated with enzalutamide versus those treated with placebo [13]. A recent post hoc analysis of the TERRAIN study suggests a higher risk for atrial fibrillation in patients with $\mathrm{MCRPC}$ taking enzalutamide (160 mg/day) versus bicalutamide (50 mg/day) [14].

Because drug-induced QT interval prolongation has been one of the most common causes of drug withdrawals or restrictions of already marketed drugs $[15,16]$, a thorough premarketing assessment of a drug's potential to cause ECG 
change or generate life-threatening arrhythmias is a regulatory requirement detailed in the International Conference on Harmonisation of Technical Requirements for Registration of Pharmaceuticals for Human Use-Harmonised Tripartite Guideline E14 (ICH E14) [17]. Therefore, the current study evaluated the effect of therapeutic doses of apalutamide (240 mg) and its active metabolite, $N$-desmethyl apalutamide, on ventricular repolarization in patients with CRPC.

In accordance with the ICH E14 guideline, a thorough QT (TQT) study ideally has a four-way crossover design, including a therapeutic dose, a supratherapeutic dose, a placebo, and a positive control. In light of the absence of a preclinical QT signal and no conclusive evidence for QTc prolongation in a previous phase I/II study, combined with the need for $\geq 8$ weeks of dosing with apalutamide to reach steady state conditions, providing 8 weeks of placebo treatment in a cancer population would be unethical. The implementation of a positive control would have required the standalone administration of a positive control (e.g., moxifloxacin) and adequate washout prior to starting apalutamide treatment, to avoid any carryover effect on the predose (Day - 1) and Cycle 1 Day 1 (C1D1) ECG assessments. Moreover, there is limited safety experience with apalutamide at a dose of $>240 \mathrm{mg}$ from previous clinical studies. Thus, an alternative multiple dose-dedicated QTc study design was chosen and customized for the oncological setting and for the PK characteristics of apalutamide.

\section{Materials and methods}

\section{Patients}

Enrolled patients were diagnosed with adenocarcinoma of the prostate and with either high-risk nmCRPC [prostate-specific antigen (PSA) doubling time $\leq 10$ months] or mCRPC. Other inclusion factors were surgical or medical castration with testosterone levels $<50 \mathrm{ng} / \mathrm{dL}$, Eastern Cooperative Oncology Group (ECOG) performance status 0 or 1 , adequate bone marrow and organ function, $\mathrm{QTcF} \leq 470 \mathrm{~ms}$, and left ventricular ejection fraction of $>45 \%$. Key exclusion criteria included known brain metastases, prior treatment with enzalutamide or apalutamide, grade $\geq 2$ electrolyte abnormalities (hypokalemia, hypocalcemia, hypomagnesemia), uncontrolled hypertension, significant cardiac function abnormalities on screening ECG, and history or evidence of certain cardiac conditions. Patients requiring concurrent therapy with medications known to be associated with QTc interval prolongation and an increased risk of torsades de pointes were excluded from the study. Per protocol, strong CYP3A4 inducers, strong CYP2C8 inducers (e.g., rifampin), and strong CYP2C8 inhibitors (e.g., gemfibrozil) were prohibited to not influence apalutamide exposure levels.

\section{Study design}

This was an open-label, multicenter, phase Ib study to investigate the effect of daily apalutamide (240 mg, orally) on ventricular repolarization (QTc). Approximately 42 patients with high-risk nmCRPC (defined as having a PSA doubling time of $\leq 10$ months) or mCRPC were planned to be enrolled to ensure that at least 38 patients completed the study.

The study consisted of screening, treatment, and followup phases. After providing informed consent, the patients entered a 28-day screening phase for determination of eligibility. If eligible, patients began the open-label treatment phase and were monitored for PK, pharmacodynamics [(PD) ECGs], and safety (including cardiac safety). Apalutamide was administered in continuous 28-day treatment cycles. The duration of the treatment phase for PD (ECG) and PK data collection was from baseline on C1D-1 until C3D1 (Day 57). Patients were allowed to continue apalutamide treatment after C3D1 until disease progression, withdrawal of consent, loss to follow-up, the occurrence of unacceptable toxicity, or loss of clinical benefit (investigator opinion). The follow-up phase for AEs lasted from discontinuation of apalutamide until 30 days after the last dose. Upon discontinuation of study drug, patients returned once for an end-of-treatment (EoT) visit $\leq 30$ days after their last dose.

\section{Pharmacodynamic (ECG) evaluations}

Patients were admitted to the study center on C1D-1 (baseline), C1D1, and C1D3 for PK/PD evaluation. Study drug intake was planned at 9:00 a.m. on C1D1 and C1D3 after overnight fasting. Continuous 12-lead ECGs were collected by a Holter monitor on C1D-1, C1D1, and C3D1 from 8:00 a.m. to 3:00 p.m. Triplicate 12-lead ECGs were obtained during a 10-min time interval at the following time points: at predose $(-0.5 \mathrm{~h})$ and at $1,2,3,4$, and $5 \mathrm{~h}$ after apalutamide administration. This 10-min timeframe began $5 \mathrm{~min}$ before and ended $5 \mathrm{~min}$ after each scheduled time point. Holter recordings were sent to a blinded, third-party, central ECG contract laboratory for 12-lead ECG selection/extraction, ECG interval measurements, and ECG interpretation.

ECG parameters measured included QRS (the onset of ventricular depolarization), PR (the period extending from the beginning of the onset of atrial depolarization until the beginning of the QRS complex), RR (with R being the point corresponding to the peak of the QRS complex of the ECG wave, and RR being the interval between successive Rs), and QT (electrical depolarization and repolarization of the ventricles). 


\section{Pharmacokinetic evaluations}

After apalutamide administration on C1D1 and C3D1, time-matched PK blood samples were collected $\leq 5 \mathrm{~min}$ after completion of the 10-min timeframe for planned ECG collection at predose $(-0.5 \mathrm{~h})$ and at $1,2,3,4$, and $5 \mathrm{~h}$ postdose. To calculate an area under the curve (AUC) value over $24 \mathrm{~h}$ after the first dose (C1D1), a 24-h PK sample was also collected during C1D2. Plasma concentrations of apalutamide and $\mathrm{N}$-desmethyl apalutamide were determined using validated liquid chromatography/tandem mass spectrometry methods. The assay consisted of protein precipitation followed by liquid chromatography with tandem mass spectrometric detection. Stable isotope-labeled internal standards were used for quantification. Chromatography was performed with a Waters Xbridge C18 column $(50 \times 2.1 \mathrm{~mm}$, $3.5 \mu \mathrm{m}$ ) using a gradient with $0.1 \%$ formic acid and acetonitrile. An API5000 mass spectrometer in the negative ion mode with a Turbo-Ionspray Interface (AB SCIEX, MA, USA) was used. Multiple reaction monitoring (MRM) transitions were from $\mathrm{m} / \mathrm{z} 476.1$ to 419.1 and from 479.1 to 419.1 for apalutamide and the internal standard, respectively. For $N$-desmethyl apalutamide and the internal standard, respectively, MRM transitions were from $\mathrm{m} / \mathrm{z} 464.1$ to 235.0 and from 469.2 to 240.1 . The quantification range was $0.0250-25.0 \mu \mathrm{g} / \mathrm{mL}$ for both analytes, and the assay performance was monitored using quality control samples. The recorded values all met the acceptance criteria.

PK parameters calculated for apalutamide and $\mathrm{N}$-desmethyl apalutamide using noncompartmental analysis included $C_{\text {max }}, t_{\text {max }}, \mathrm{AUC}$ from time 0 to $24 \mathrm{~h}$ after dosing $\left(\mathrm{AUC}_{24 \mathrm{~h}}\right)$, and the minimum observed plasma concentration $\left(C_{\text {min }}\right)$. Additionally, the accumulation index (AI), metabolite:parent drug ratio, corrected for molecular weight (MPR; for $\mathrm{N}$-desmethyl apalutamide only), and peak/trough ratio at steady state (PTR) were also calculated.

The PK/PD data collection sought to determine the potential relationship between change from baseline in QTc $(\Delta \mathrm{QTc})$ and the plasma concentrations of apalutamide and $\mathrm{N}$-desmethyl apalutamide. The measured QT data were corrected for heart rate using Fridericia (QTcF) [18], Bazett (QTcB), and a study-specific correction Power (QTcP) correction method. The correlation between QTcF and RR was not significant, with a slope of 0.031 and a $90 \%$ confidence interval $(\mathrm{CI})(-0.01$ to 0.07$)$ that included zero. A similar analysis with the QTcP method also showed no statistically significant relationship between QTc and RR, whereas with the QTcB method a statistically significant relationship between QTc and RR was observed. Overall, these analyses support the use of the QTcF method as the primary correction method; thus, only QTcF data are reported herein. Baseline was defined as the mean QTc values of the triplicate ECG measurements taken at baseline. These baseline QTc values were time-matched with those on C1D1 and C3D1. The $\Delta$ QTc was calculated at each time point. The primary endpoint was the maximum mean $\Delta \mathrm{QTC}$ on C3D1, which was estimated by the mean $\Delta$ QTc at around $t_{\max }$ (i.e., steady state). The duration of PD and PK assessments during the treatment phase was from baseline until C3D1.

\section{Safety evaluations}

Patients were monitored for safety during the screening, treatment, and follow-up phases until 30 days after the last dose of study drug. The safety evaluations included AE reporting, clinical laboratory safety evaluations, ECGs, multigated acquisition scan, or echocardiogram (screening only for determination of left ventricular ejection fraction), ECOG performance status scores, vital signs, and physical examination. For patients who remained in the study after three cycles of apalutamide treatment, collection of AEs was limited to serious AEs (SAEs) and grade $\geq 3$ AEs. Patients were followed for disease progression as clinically indicated per institutional guidelines, which could include PSA monitoring or imaging collected at the discretion of the investigator. The safety population included all patients who received at least one dose of apalutamide.

\section{Statistical analysis}

The clinical cutoff for the statistical analysis of the study was defined when the last patient enrolled had completed the C3D1 (Day 57) assessments. For the statistical analysis based on the primary correction method (QTcF), the mean changes from baseline $(\Delta \mathrm{QTcF})$ at each time point were summarized [mean, standard deviation (SD), median and range, two-sided $90 \% \mathrm{CI}$ ]. The primary endpoint analysis focused on the maximum mean $\Delta \mathrm{QTcF}$ at $\mathrm{C} 3 \mathrm{D} 1$, which was estimated by the mean QTcF change at around $t_{\max }$, the time when $C_{\max }$ was reached. The mean $\Delta \mathrm{QTcF}( \pm \mathrm{SD})$ over time was plotted. For each treatment and time point of measurement, HR, QRS, PR, and RR intervals, as well as the change from baseline in HR, QRS, PR, and RR $(\Delta \mathrm{HR}, \Delta \mathrm{QRS}, \Delta \mathrm{PR}$, $\Delta R R$ ), were summarized using descriptive statistics (mean, $\mathrm{SD}$, median, range, and $90 \% \mathrm{CI}$ ).

Individual plasma concentrations for apalutamide and its active metabolite $N$-desmethyl apalutamide were tabulated with descriptive statistics (including arithmetic mean, SD, coefficient of variation, median, minimum, and maximum) at each sampling time point for each visit. Individual and mean plasma concentration-time profiles were plotted.

A linear mixed-effects model was fitted to the $\Delta \mathrm{QTc}$ data from C1D1 and C3D1 with either parent or metabolite concentration as a predictor and patient as a random effect. On the basis of these relationships, the predicted population average $\Delta \mathrm{QTC}$ and its corresponding upper $90 \%$ two-sided 
CI bound were computed at the mean $C_{\max }$ of apalutamide and $\mathrm{N}$-desmethyl apalutamide, or other concentrations of interest.

\section{Results}

\section{Patient and disease characteristics}

At the time of clinical cutoff, 45 men enrolled at five study centers received at least one dose of apalutamide and were included in the safety analysis set. At study entry, the majority $(97.8 \%)$ of patients had mCRPC. One patient had highrisk nmCRPC. Forty three of the 45 patients were considered study completers (defined as having completed C3D1 ECG collection and PK sampling procedures in the presence of adequate compliance with intake of the study drug during Cycles 1 and 2) and were included in the primary analysis set. Median age at study entry was 67 years (range 52-86 years) (Table 1). All patients had received therapy for prostate cancer prior to study entry in addition to ADT or surgical castration; the most commonly prescribed prior therapies were bicalutamide $(89 \%)$, abiraterone acetate $(42 \%)$, and docetaxel (38\%) (Table 1). Overall, study participants were largely compliant with avoidance of prohibited

Table 1 Patient and disease characteristics

\begin{tabular}{lc}
\hline Baseline characteristic & Total $(N=45)$ \\
\hline Median age, year (range) & $67(52-86)$ \\
Race, $n(\%)$ & \\
White & $42(93)$ \\
Black or African American & $3(7)$ \\
Median weight, kg (range) & $80(50-135)$ \\
Median time from initial diagnosis, mo (range) & $68.2(3.9-280.3)$ \\
Extent of disease, $n(\%)$ & \\
Bone & $40(91)$ \\
Soft tissue or node & $14(32)$ \\
Liver & $2(4)$ \\
Lung & $1(2)$ \\
Other & $3(7)$ \\
None & $1(2)$ \\
ECOG PS, $n(\%)$ & \\
0 & $27(60)$ \\
1 & $18(40)$ \\
Median testosterone, nM (range) & $0.85(0.07-1.63)$ \\
Prior therapy, $n(\%)$ & $45(100)$ \\
Bicalutamide & $40(89)$ \\
Abiraterone acetate & $19(42)$ \\
Docetaxel & $17(38)$ \\
Cabazitaxel & $11(24)$ \\
\hline
\end{tabular}

ECOG PS Eastern Cooperative Oncology Group performance status medications that could influence apalutamide or $N$-desmethyl apalutamide PK.

\section{Patient disposition}

At the time of clinical cutoff, the median treatment duration was 5 months (range 2-8 months); $82 \%$ of patients had received treatment for $\geq 3$ months. Furthermore, 13 of 45 patients had discontinued treatment. Treatment was discontinued due to progressive disease in 12 patients, and one patient withdrew consent.

\section{Primary endpoint}

A total of 831 evaluable ECGs were reviewed in this study, out of 855 expected ECG extractions. For each QTc correction method, the relation between QTc and RR at baseline was evaluated graphically by plotting the logarithm of baseline QTc values against the logarithm of the corresponding RR intervals. This analysis supported the QTcF method being used as the primary correction method. The primary endpoint was maximum mean $\Delta \mathrm{QTCF}$ on $\mathrm{C} 3 \mathrm{D} 1$. There was no notable difference in QTcF intervals between baseline (Day - 1) and after the first dosing (C1D1). For all the corresponding time points on C3D1, there were mean increases from baseline in QTcF, but no obvious time-related trends over the course of the day. The least squares mean increases from baseline on C3D1 ranged from 8.0 to $12.4 \mathrm{~ms}$ (Table 2). The least squares mean (standard error) QTcF change at $t_{\max }$ on C1D1 and on C3D1 was +1.9 (1.6) ms and +12.4 (2.1) ms, respectively. The upper limit of the $90 \% \mathrm{CI}$ of the least squares mean baseline corrected QTcF change at each postdose time point was below $10 \mathrm{~ms}$ for C1D1 (maximum of upper limits $=4.5 \mathrm{~ms}$ ) and above $10 \mathrm{~ms}$, for C3D1 (maximum of upper limits $=16.0 \mathrm{~ms}$ ).

Patients with QTcF intervals exceeding the threshold values of 450,480 , and $500 \mathrm{~ms}$ are summarized in Table 3. Twelve patients had QTcF value $>450$ and $\leq 480 \mathrm{~ms}$ at baseline while the number increased to 20 patients on $\mathrm{C} 3 \mathrm{D} 1$. One patient had a $\mathrm{QTcF}$ interval $>480$ and $\leq 500 \mathrm{~ms}$ at $1 \mathrm{~h}$ postdose on C3D1; this same patient also had a predose (C1D1, at $-1 \mathrm{~h}) \mathrm{QTcF}$ value of $469.3 \mathrm{~ms}$. No QTcF intervals > $500 \mathrm{~ms}$ were recorded. Numbers of patients with a QTcF interval change from baseline exceeding the threshold values of 30 or $60 \mathrm{~ms}$ are also summarized in Table 3. Two patients on C1D1 and nine patients on $\mathrm{C} 3 \mathrm{D} 1 \mathrm{had}$ a QTcF interval change from baseline of $>30$ but $\leq 60 \mathrm{~ms}$. Among the patients with QTcF interval changes from baseline of $>30 \mathrm{~ms}$ but $\leq 60 \mathrm{~ms}$, no association was observed with the presence of underlying electrolyte abnormalities or significant cardiac medical history. One patient had a QTcF interval change from baseline of $60.4 \mathrm{~ms}$ at $1 \mathrm{~h}$ postdose on C3D1 
Table 2 Mean $\Delta$ QTcF over time after single dose and steady state

\begin{tabular}{|c|c|c|c|c|c|c|}
\hline & \multicolumn{3}{|c|}{ Absolute QTcF interval $^{\mathrm{a}}$, ms } & \multicolumn{3}{|c|}{$\Delta \mathrm{QTcF}\left(\mathrm{LS}\right.$ mean) interval ${ }^{\mathrm{b}}, \mathrm{ms}$} \\
\hline & $N$ & Mean (SD) & $95 \% \mathrm{CI}$ & $N$ & Mean (SE) & $90 \% \mathrm{CI}$ \\
\hline \multicolumn{7}{|l|}{ C1D1 } \\
\hline Predose & 43 & $428.7(13.5)$ & $(424.5-432.9)$ & 43 & $-0.7(1.59)$ & -3.4 to 1.9 \\
\hline $1 \mathrm{~h}$ & 42 & $430.1(14.5)$ & $(425.6-434.6)$ & 41 & $-0.4(1.62)$ & -3.1 to 2.3 \\
\hline $2 \mathrm{~h}$ & 43 & $432.4(15.2)$ & $(427.7-437.0)$ & 42 & $1.9(1.61)$ & -0.8 to 4.5 \\
\hline $3 \mathrm{~h}$ & 43 & 424.7 (13.9) & $(420.4-429.0)$ & 42 & $-3.1(1.61)$ & -5.8 to -0.4 \\
\hline $4 \mathrm{~h}$ & 43 & $425.7(14.5)$ & $(421.3-430.2)$ & 42 & $-2.1(1.61)$ & -4.8 to 0.6 \\
\hline $5 \mathrm{~h}$ & 43 & $422.4(15.9)$ & $(417.5-427.3)$ & 41 & $-5.5(1.62)$ & -8.2 to -2.8 \\
\hline \multicolumn{7}{|l|}{ C3D1 } \\
\hline Predose & 42 & $441.6(16.8)$ & $(436.3-446.8)$ & 42 & $12.0(2.14)$ & $8.4-15.5$ \\
\hline $1 \mathrm{~h}$ & 42 & $442.7(18.6)$ & $(436.9-448.5)$ & 41 & $12.3(2.16)$ & $8.7-15.9$ \\
\hline $2 \mathrm{~h}$ & 43 & $442.9(16.4)$ & $(437.8-447.9)$ & 42 & $12.4(2.15)$ & $8.8-16.0$ \\
\hline $3 \mathrm{~h}$ & 42 & $439.3(15.7)$ & $(434.4-444.2)$ & 41 & $10.9(2.15)$ & $7.3-14.5$ \\
\hline $4 \mathrm{~h}$ & 42 & $436.5(14.2)$ & $(432.0-440.9)$ & 41 & $8.2(2.15)$ & $4.6-11.8$ \\
\hline $5 \mathrm{~h}$ & 42 & $436.0(16.3)$ & $(430.9-441.1)$ & 40 & $8.0(2.16)$ & $4.4-11.6$ \\
\hline
\end{tabular}

$C I$ confidence interval, $L S$ least squares, $S D$ standard deviation, $S E$ standard error, $C 1 D-1$ Cycle 1 Day - 1, C1D1 Cycle 1 Day 1, C3D1 Cycle 3 Day 1

${ }^{\text {a }}$ The time-matched baseline is defined as the mean values of the triplicate electrocardiographic measurements taken on C1D-1, at the time points matching with those on C1D1 and C3D1

${ }^{\mathrm{b}} \mathrm{A}$ repeated-measures mixed model was used with time point and baseline value of QTc as fixed effect, and patient as a random effect

\begin{tabular}{lllll}
\hline & $\begin{array}{l}\text { Baseline } \\
(N=43)\end{array}$ & $\begin{array}{l}\text { C1D1 } \\
(N=43)\end{array}$ & $\begin{array}{l}\text { C3D1 } \\
(N=43)\end{array}$ & $\begin{array}{l}\text { Total } \\
\text { (C1D1+C3D1) } \\
(N=43)\end{array}$ \\
\hline \begin{tabular}{l} 
Pre- or post-apalutamide \\
\multicolumn{2}{l}{ QTcF $>450 \mathrm{~ms}, n(\%)^{\mathrm{a}}$}
\end{tabular} & & & \\
$>450$ to $\leq 480 \mathrm{~ms}$ & $12(28)$ & $6(14)$ & $20(47)$ & $20(47)$ \\
$>480$ to $\leq 500 \mathrm{~ms}$ & 0 & 0 & $1(2)$ & $1(2)$ \\
$>500 \mathrm{~ms}$ & 0 & 0 & 0 & 0 \\
$\begin{array}{l}\text { QTcF increase from baseline }>30 \mathrm{~ms}, n(\%)^{\mathrm{b}} \\
>30 \text { to } \leq 60 \mathrm{~ms}\end{array}-$ & $2(5)$ & $9(21)$ & $9(21)$ \\
$>60 \mathrm{~ms}$ & - & 0 & $1(2)$ & $1(2)$ \\
\hline
\end{tabular}

$C 1 D-1$ Cycle 1 Day - 1, C1D1 Cycle 1 Day, C3D1 Cycle 3 Day 1

${ }^{a}$ Percentages are calculated with the number of patients in primary analysis as denominators and only the worst value for a patient is presented; the C1D1 predose measurement and C1D-1 measurement are considered as baseline

${ }^{\mathrm{b}}$ Percentages are calculated with the number of patients in primary analysis as denominators; time-matched baseline is defined as the mean values of the triplicate electrocardiographic measurements taken on C1D-1 (including predose), at the time points matching with those on C1D1 (including predose) and C3D1 (including predose)
( $455.7 \mathrm{~ms}$ ). The two patients with QTcF $>480 \mathrm{~ms}$ or with QTcF increase of $>60 \mathrm{~ms}$ from baseline did not have a significant cardiac medical history and did not use any concomitant medications with a liability for QTc prolongation. The patient with QTcF $>480 \mathrm{~ms}$ on C3D1 showed grade 1 hypocalcemia at baseline and $\mathrm{C} 3 \mathrm{D} 1$, but potassium and magnesium levels were normal.

\section{Secondary endpoints}

For all time points on C1D1, mean heart rate was increased from baseline (Table 4), without obvious time-related trends over the course of the day. The mean increases from baseline on C1D1 ranged from 0.1 to $2.5 \mathrm{bpm}$. For all time points on $\mathrm{C} 3 \mathrm{D} 1$, there were mean decreases from baseline in HR, but with no obvious time-related trends over the course of 
Table 4 Change from baseline in heart rate, RR interval, PR interval, and QRS interval at C1D1 and C3D1

\begin{tabular}{|c|c|c|c|}
\hline & $N$ & Mean \pm SD & $90 \% \mathrm{CI}$ \\
\hline \multicolumn{4}{|c|}{ Heart rate, bpm } \\
\hline \multicolumn{4}{|c|}{ C1D1 } \\
\hline Predose & 43 & $0.6 \pm 5.83$ & -0.9 to 2.1 \\
\hline $1 \mathrm{~h}$ & 41 & $0.7 \pm 5.96$ & -0.9 to 2.3 \\
\hline $2 \mathrm{~h}$ & 42 & $2.4 \pm 13.11$ & -1.0 to 5.8 \\
\hline $3 \mathrm{~h}$ & 42 & $2.5 \pm 11.97$ & -0.6 to 5.7 \\
\hline $4 \mathrm{~h}$ & 42 & $0.1 \pm 13.26$ & -3.4 to 3.5 \\
\hline $5 \mathrm{~h}$ & 41 & $2.2 \pm 6.01$ & 0.6 to 3.8 \\
\hline \multicolumn{4}{|l|}{ C3D1 } \\
\hline Predose & 42 & $-2.5 \pm 6.09$ & -4.0 to -0.9 \\
\hline $1 \mathrm{~h}$ & 41 & $-1.7 \pm 5.93$ & -3.2 to -0.1 \\
\hline $2 \mathrm{~h}$ & 42 & $-3.4 \pm 6.60$ & -5.1 to -1.7 \\
\hline $3 \mathrm{~h}$ & 41 & $-1.3 \pm 13.69$ & -4.9 to 2.3 \\
\hline $4 \mathrm{~h}$ & 41 & $-3.5 \pm 14.82$ & -7.4 to 0.4 \\
\hline $5 \mathrm{~h}$ & 40 & $-0.4 \pm 7.17$ & -2.3 to 1.5 \\
\hline \multicolumn{4}{|c|}{ RR interval, ms } \\
\hline \multicolumn{4}{|c|}{ C1D1 } \\
\hline Predose & 43 & $-7.5 \pm 89.97$ & -30.6 to 15.5 \\
\hline $1 \mathrm{~h}$ & 41 & $-13.3 \pm 92.29$ & -37.6 to 11.0 \\
\hline $2 \mathrm{~h}$ & 42 & $-16.1 \pm 92.55$ & -40.1 to 8.0 \\
\hline $3 \mathrm{~h}$ & 42 & $-16.0 \pm 83.55$ & -37.7 to 5.7 \\
\hline $4 \mathrm{~h}$ & 42 & $-6.4 \pm 86.57$ & -28.9 to 16.1 \\
\hline $5 \mathrm{~h}$ & 41 & $-21.5 \pm 63.13$ & -38.1 to 4.9 \\
\hline \multicolumn{4}{|l|}{ C3D1 } \\
\hline Predose & 42 & $39.0 \pm 89.10$ & 15.8 to 62.1 \\
\hline $1 \mathrm{~h}$ & 41 & $27.5 \pm 84.93$ & 5.2 to 49.9 \\
\hline $2 \mathrm{~h}$ & 42 & $41.0 \pm 77.88$ & 20.8 to 61.2 \\
\hline $3 \mathrm{~h}$ & 41 & $33.2 \pm 143.13$ & -4.5 to 70.8 \\
\hline $4 \mathrm{~h}$ & 41 & $36.4 \pm 141.19$ & -0.7 to 73.5 \\
\hline $5 \mathrm{~h}$ & 40 & $6.3 \pm 79.87$ & -15.0 to 27.6 \\
\hline \multicolumn{4}{|c|}{ PR interval, ms } \\
\hline \multicolumn{4}{|c|}{ C1D1 } \\
\hline Predose & 43 & $2.6 \pm 12.34$ & -0.5 to 5.8 \\
\hline $1 \mathrm{~h}$ & 41 & $1.9 \pm 13.01$ & -1.6 to 5.3 \\
\hline $2 \mathrm{~h}$ & 41 & $-0.7 \pm 10.12$ & -3.4 to 2.0 \\
\hline $3 \mathrm{~h}$ & 41 & $-1.1 \pm 9.78$ & -3.7 to 1.5 \\
\hline $4 \mathrm{~h}$ & 41 & $-1.8 \pm 9.51$ & -4.3 to 0.7 \\
\hline $5 \mathrm{~h}$ & 41 & $-0.3 \pm 6.31$ & -2.0 to 1.4 \\
\hline \multicolumn{4}{|l|}{ C3D1 } \\
\hline Predose & 42 & $2.2 \pm 13.70$ & -1.3 to 5.8 \\
\hline $1 \mathrm{~h}$ & 41 & $0.1 \pm 17.29$ & -4.4 to 4.7 \\
\hline $2 \mathrm{~h}$ & 42 & $1.6 \pm 11.38$ & -1.4 to 4.5 \\
\hline $3 \mathrm{~h}$ & 40 & $-0.9 \pm 15.72$ & -5.1 to 3.3 \\
\hline $4 \mathrm{~h}$ & 40 & $-2.7 \pm 14.82$ & -6.6 to 1.3 \\
\hline $5 \mathrm{~h}$ & 40 & $0.6 \pm 8.54$ & -1.7 to 2.8 \\
\hline \multicolumn{4}{|c|}{ QRS interval, ms } \\
\hline \multicolumn{4}{|l|}{ C1D1 } \\
\hline Predose & 43 & $0.5 \pm 3.36$ & -0.4 to 1.4 \\
\hline $1 \mathrm{~h}$ & 41 & $0.9 \pm 3.35$ & 0.0 to 1.8 \\
\hline
\end{tabular}

Table 4 (continued)

\begin{tabular}{cccl}
\hline & $N$ & Mean \pm SD & $90 \%$ CI \\
\hline $2 \mathrm{~h}$ & 42 & $0.5 \pm 3.97$ & -0.5 to 1.6 \\
$3 \mathrm{~h}$ & 42 & $0.8 \pm 3.58$ & -0.2 to 1.7 \\
$4 \mathrm{~h}$ & 42 & $0.9 \pm 3.00$ & 0.1 to 1.6 \\
$5 \mathrm{~h}$ & 41 & $0.5 \pm 3.53$ & -0.4 to 1.5 \\
$\mathrm{C} 3 \mathrm{D} 1$ & & & \\
Predose & 42 & $2.4 \pm 4.53$ & $1.2-3.6$ \\
$1 \mathrm{~h}$ & 41 & $1.6 \pm 5.05$ & $0.3-2.9$ \\
$2 \mathrm{~h}$ & 42 & $1.9 \pm 4.91$ & $0.7-3.2$ \\
$3 \mathrm{~h}$ & 41 & $2.2 \pm 4.88$ & $0.9-3.4$ \\
$4 \mathrm{~h}$ & 41 & $1.6 \pm 5.07$ & $0.3-3.0$ \\
$5 \mathrm{~h}$ & 40 & $2.3 \pm 4.71$ & $1.1-3.6$ \\
\hline
\end{tabular}

$S D$ standard deviation, $C I$ confidence interval, C1D-1 Cycle 1 Day - 1, C1D1 Cycle 1 Day 1, C3D1 Cycle 3 Day 1

the day. The mean decreases from baseline on C3D1 ranged from -0.4 to $-3.5 \mathrm{bpm}$. The number of patients with any HR [ $>100 \mathrm{bpm}(n=3)$ or $<50 \mathrm{bpm}(n=2)]$ was similar at baseline and on C1D1 and C3D1 (data not shown). Apalutamide did not have a clinically significant effect on HR.

For all time points on C1D1, there were mean decreases from baseline in RR interval but with no obvious timerelated trends over the course of the day (Table 4). The decreases from baseline on C1D1 ranged from -6.4 to $-21.5 \mathrm{~ms}$. For all time points on C3D1, there were mean increases from baseline in RR interval, also with no obvious time-related trends over the course of the day. The increases from baseline on C3D1 ranged from 6.3 to $41.0 \mathrm{~ms}$. The observations on the RR interval are inversely correlated with the observations on HR interval.

For mean PR interval over time compared with baseline, no obvious time-related trends were noted over the course of C1D1 or C3D1 (Table 4). The incidence count and percentage of patients with any PR interval $>200 \mathrm{~ms}$ by study day and by time point was similar at baseline and on C1D1 or C3D1 (data not shown). No effect of apalutamide on the length of the PR interval was apparent. For all time points on C1D1 and C3D1, mean increases were observed from baseline in the QRS interval (Table 4). The mean increases from baseline on C1D1 ranged from 0.5 to $0.9 \mathrm{~ms}$ and on C3D1 from 1.6 to $2.4 \mathrm{~ms}$. No patients had a QRS interval $>110 \mathrm{~ms}$ at baseline or on C1D1. QRS intervals $>110 \mathrm{~ms}$ but $\leq 115 \mathrm{~ms}$ on C3D1 were recorded in three patients. The largest mean change (increase) in QRS duration from baseline was $2.4 \mathrm{~ms}$ on C3D1 at predose. Overall, no clinically relevant effects of apalutamide on the QRS interval were observed.

T-wave morphology was monitored, and the number of patients with flat, inverted, or biphasic T-waves was similar on pretreatment and post-treatment days. For most patients 
with T-wave abnormalities observed during the treatment phase, these observations were also noted on predose ECG before apalutamide administration. De novo T-wave abnormalities were observed in three patients $(7 \%)$, which were absent at baseline, and no QTcF prolongation $\geq 480 \mathrm{~ms}$ was observed in these three patients. Apalutamide treatment did not have an apparent association with the appearance or worsening of T-wave abnormalities, and no U-waves were observed in any patient.

Mean plasma concentrations over time for apalutamide and $N$-desmethyl apalutamide are shown in Fig. 1a, b. Repeated once-daily administration of 240-mg apalutamide under fasted conditions resulted in a three- and fivefold increase of $C_{\max }$ and $\mathrm{AUC}_{24 \mathrm{~h}}$, respectively, when comparing apalutamide systemic exposure between C1D1 and C3D1 (Table 5). Median $t_{\max }$ was reached at approximately $2 \mathrm{~h}$ post dose on C1D1 and C3D1. At steady state (C3D1), the active metabolite $\mathrm{N}$-desmethyl apalutamide exhibited a flat PK profile with a mean PTR of $127 \%$. The MPRs for $C_{\max }$ and $\mathrm{AUC}_{24 \mathrm{~h}}$ were

a

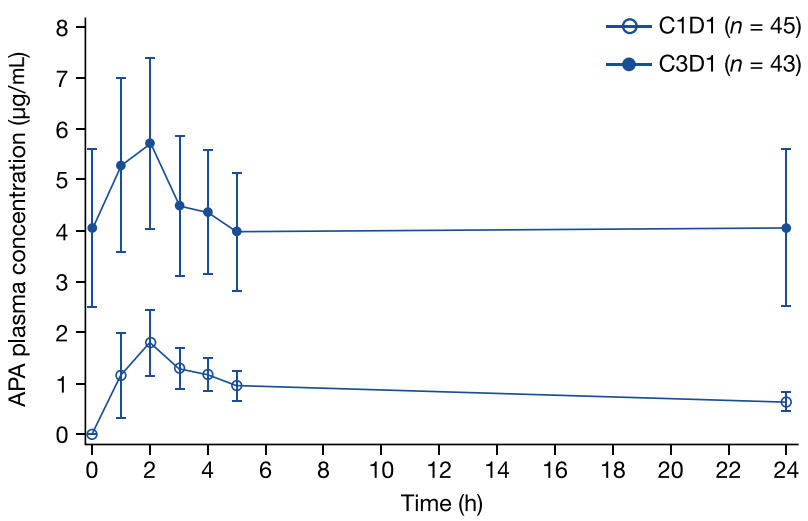

C

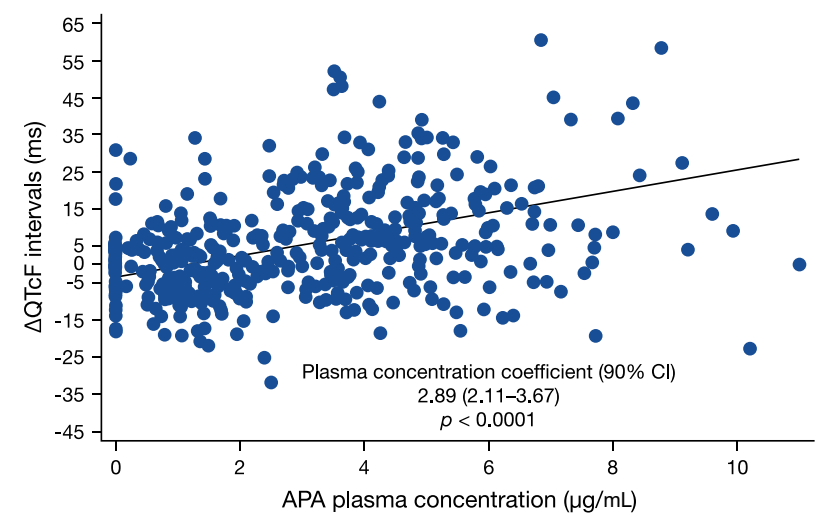

Fig. 1 Plasma concentration of apalutamide and $N$-desmethyl apalutamide and their association with QTcF: a mean plasma concentration-time profiles of apalutamide after administration of $240 \mathrm{mg}$ apalutamide on C1D1 and C3D1; $\mathbf{b}$ mean plasma concentration-time profiles of $\mathrm{N}$-desmethyl apalutamide after administration of $240 \mathrm{mg}$
$105 \pm 21$ and $133 \pm 28 \%$, respectively (Table 5). A significant correlation was observed between the change in QTcF from baseline and the concentration of apalutamide (slope estimate, 2.89; 90\% CI, 2.11-3.67; $p<0.0001$ ) (Fig. 1c). The predicted $\Delta \mathrm{QTcF}(90 \% \mathrm{CI})$ at a mean $C_{\max }$ of $5.95 \mu \mathrm{g} / \mathrm{mL}$ was $13.8 \mathrm{~ms}$ (9.77-17.85). Likewise, a significant correlation was observed between the change in QTcF from baseline and the concentration of $\mathrm{N}$-desmethyl apalutamide (slope estimate, $2.28 ; 90 \%$ CI 1.70-2.85; $p<0.0001$ ) (Fig. 1d). For instance, on C3D1 the predicted $\triangle \mathrm{QTcF}(90 \% \mathrm{CI})$ at a mean steady state $C_{\max }$ of $5.84 \mu \mathrm{g} / \mathrm{mL}$ was $12.0 \mathrm{~ms}(8.58-15.38)$.

\section{Safety}

Dose modifications were allowed for toxicity attributed to apalutamide, and re-escalation was permitted if first discussed with and approved by the sponsor. The majority of patients had neither dose reduction (42 patients, 93\%) nor dose interruption (38 patients, $84 \%$ ). There were no dose

b

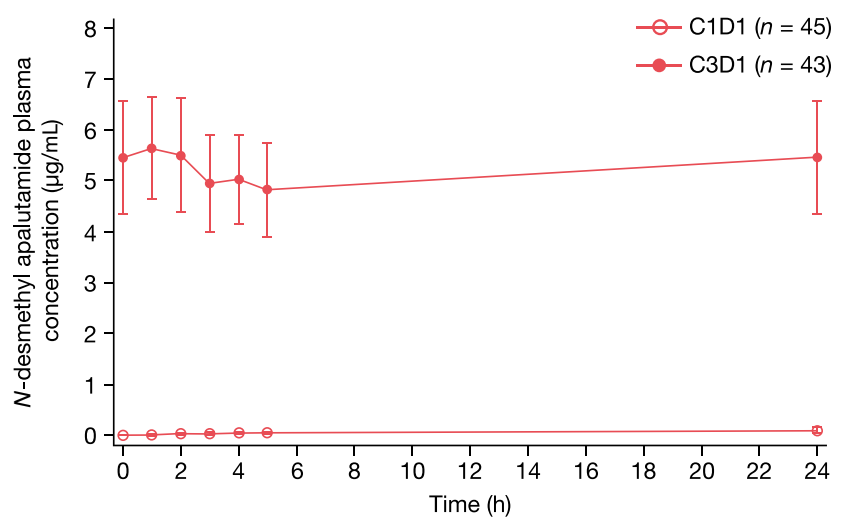

d

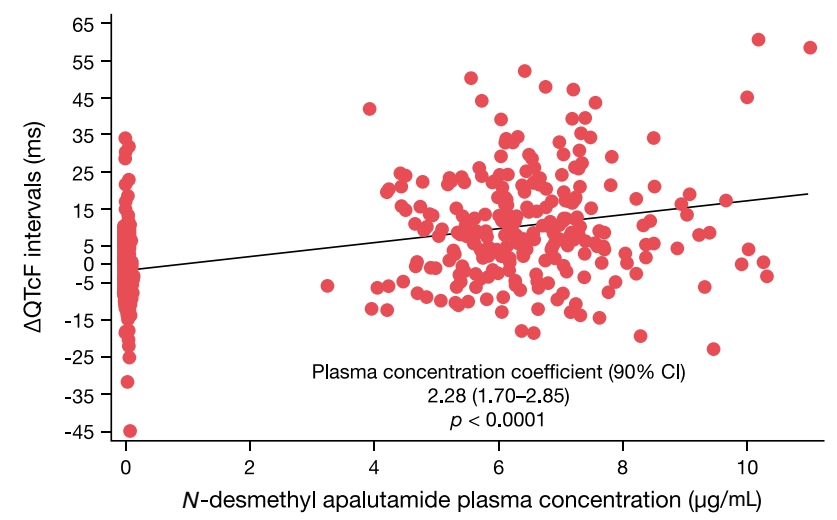

apalutamide on C1D1 and C3D1; c scatter plot of the relationship between $\Delta \mathrm{QTcF}$ and plasma concentration of apalutamide; $\mathbf{d}$ scatter plot of the relationship between $\Delta \mathrm{QTcF}$ and plasma concentration of $\mathrm{N}$-desmethyl apalutamide 
Table 5 Pharmacokinetics of apalutamide and $\mathrm{N}$-desmethyl apalutamide

\begin{tabular}{|c|c|c|c|c|}
\hline \multirow[t]{2}{*}{ Parameter $^{\mathrm{a}}$} & \multicolumn{2}{|l|}{ Apalutamide } & \multicolumn{2}{|c|}{$N$-desmethyl apalutamide } \\
\hline & C1D1 $(N=45)$ & C3D1 $(N=43)$ & C1D1 $(N=45)$ & C3D1 $(N=43)$ \\
\hline$C_{\max }, \mu \mathrm{g} / \mathrm{mL}$ & $2.06(0.58)$ & $5.95(1.66)$ & $0.092(0.057)$ & $5.85(1.04)$ \\
\hline$t_{\max }, \mathrm{h}$ & $2.12(1.08-5.10)$ & $2.10(1.00-4.17)$ & $24.00(4.10-24.58)$ & $1.10(0.00-4.17)$ \\
\hline $\mathrm{AUC}_{24 \mathrm{~h}}, \mu \mathrm{g} \cdot \mathrm{h} / \mathrm{mL}$ & $21.1(4.93)$ & $100(31.6)$ & $1.41(0.79)$ & $124(23.0)$ \\
\hline$C_{\min }, \mu \mathrm{g} / \mathrm{mL}$ & - & $3.72(1.19)$ & - & $4.66(0.90)$ \\
\hline PTR, $\%$ & - & $163(24.7)$ & - & $127(13.3)$ \\
\hline $\mathrm{AI}_{(C \max )}$ & - & $3.09(1.26)$ & - & $82.1(50.5)$ \\
\hline $\mathrm{AI}_{(\mathrm{AUC} 24 \mathrm{~h})}$ & - & $4.95(1.69)$ & - & $122(108)$ \\
\hline MPR $C_{\max }, \%(\mathrm{SD})$ & - & - & - & $105(20.8)$ \\
\hline MPR AUC $24 \mathrm{~h}, \%(\mathrm{SD})$ & - & - & - & $133(28.0)$ \\
\hline
\end{tabular}

C1D1 Cycle 1 Day 1, C3D1 Cycle 3 Day 1, $C_{\max }$ maximum observed plasma concentration, $t_{\max }$ time to $C_{\max }, A U C_{24 h}$ AUC from time 0 to $24 \mathrm{~h}$ after dosing, $C_{\text {min }}$ minimum observed plasma concentration, PTR peak/trough ratio at steady state, $A I$ accumulation index, $M P R$ metabolite:parent drug ratio corrected for molecular weight

${ }^{a}$ All values are presented as the mean (SD) except for $t_{\max }$, which is presented as the median (range), or as otherwise noted re-escalations after initial dose reduction. Drug-related toxicities leading to temporary dose interruption included grade 3 diarrhea and aspartate transaminase/alanine transaminase increase and grade 2 fatigue. Two patients required a dose reduction due to fatigue. Thirteen $(29 \%)$ patients discontinued treatment, with 12 of those discontinuing due to progressive disease and one due to withdrawal of consent (no discontinuations were due to AEs).

Thirty-seven $(82 \%)$ patients experienced at least one treatment-emergent AE (TEAE), most of which were grades $1-2$. The most commonly reported TEAEs $(\geq 10 \%$ of patients) were fatigue $(40 \%)$, decreased appetite $(24 \%)$, back pain (16\%), diarrhea and dyspnea (13\% each), rash/ erythema (13\%), and constipation and nausea (11\% each). No treatment-emergent seizures were reported. The AEs recorded in this study were consistent with those observed in other published apalutamide studies [6, 7, 19, 20]. Grade 3 TEAEs were reported in eight (18\%) patients and grade 4 AEs in two (4\%) patients. Grade 3 TEAEs reported in $>1$ patient were anemia ( 3 patients, $7 \%$ ) and back pain (2 patients, 4\%); these grade 3 TEAEs were not considered related to apalutamide treatment. Three patients reported grade 3 toxicities considered possibly or probably related to apalutamide, including fatigue, diarrhea, and aspartate transaminase/alanine transaminase increase. In one patient, grade 3 cardiac failure was reported on study Day 45 and was not considered related to apalutamide treatment. Grade 3 nervous system disorder and spinal cord compression were reported in two patients, were not considered related to apalutamide treatment, and occurred in an overall context of worsening vertebral metastatic disease. One patient experienced grade 4 thrombocytopenia, which was not considered related to apalutamide treatment and occurred in the context of progressive disease. Another patient had grade 4 neutropenia not considered related to apalutamide treatment that occurred while the patient took sulfamethoxazole plus trimethoprim for a bladder infection.

Five patients experienced $\geq 1 \mathrm{SAE}$, but none were considered related to apalutamide treatment; SAEs were grades 1-3, except for a grade $4 \mathrm{SAE}$ of general health deterioration in one patient, who subsequently died from progressive disease. One patient experienced a grade $3 \mathrm{SAE}$ of medullary compression, which was considered not related to apalutamide treatment and likely related to underlying metastatic disease in the vertebra. Another patient with bone metastases experienced a grade $3 \mathrm{SAE}$ of progressive lower back pain, which was attributed to the magnetic resonance imaging-verified metastatic disease in the pelvis. One patient experienced multiple SAEs: grade 2 hypercalcemia, grade 3 jaw necrosis, grade 3 pain left hip, and grade 3 neurologic deficit due to spinal cord compression resulting from bone metastases; none were considered related to apalutamide treatment. The final patient, a 70-year-old man with a history of hypertension, mitral valve prolapse, and type 2 diabetes, experienced multiple SAEs, including grade 3 lower back pain, grade 2 infection, and grade 2 delirium, none of which were considered related to apalutamide treatment. This patient also experienced a grade $3 \mathrm{SAE}$ of heart failure caused by de novo atrial fibrillation that was not considered related to apalutamide treatment.

Laboratory values were collected over time (data not shown). Most patients had occasional changes in serum chemistry and some hematologic abnormalities, the majority of which were grade 0-2 in severity. Elevated thyroidstimulating hormone (TSH) levels above the upper limit of normal during the study were observed in 13 patients $(29 \%)$, 
were usually limited in magnitude and, in the majority of cases, thyroid hormone levels stayed within normal limits. In two patients (4\%), significant TSH elevations were observed in combination with a decrease in thyroid hormone levels. One of these two patients had a medical history of hypothyroidism and required an increase in thyroid supplementation therapy in the course of the study.

\section{Discussion}

These data from an open-label, multicenter, phase Ib dedicated QT/QTc study that investigated the effect of apalutamide on ventricular repolarization and other ECG parameters confirm the absence of major effects from apalutamide on the QTc interval in men with CRPC. This modified QTc study, tailored to the oncologic setting and taking into account the PK characteristics of apalutamide, was rigorously executed with time-matched ECG and PK sample collection and central blinded ECG interval measurement and interpretation. Across all time points at steady state, the baseline-adjusted QTc intervals and the upper bounds of their associated $90 \%$ CIs were $\leq 20$ ms following 240 $\mathrm{mg}$ once-daily doses of apalutamide. Consistent with the primary endpoint results, categorical analysis of absolute QTcF values revealed a slightly higher incidence of QTcF readings $>450$ but $\leq 480 \mathrm{~ms}$ on C3D1 compared with baseline and C1D1. These results indicate that the QTc increases observed with apalutamide become apparent at steady state (after minimally 4 weeks) but not after the first dose, likely because of an accumulation of apalutamide with repeat dosing.

There were two outliers with a larger QTc prolongation. One patient with a QTcF interval $>480$ and $\leq 500 \mathrm{~ms}$ also had a higher predose QTcF value $(469.3 \mathrm{~ms})$ and had a concurrent C3D1 observation of grade 1 hypocalcemia. A second patient had a large absolute $\Delta \mathrm{QTcF}(60.4 \mathrm{~ms})$ at $1 \mathrm{~h}$ post dose on C3D1 (QTcF $455.7 \mathrm{~ms}$ ), which may have been related to exposure to apalutamide $(6.84 \mu \mathrm{g} / \mathrm{mL})$ or $N$-desmethyl apalutamide $(8.21 \mu \mathrm{g} / \mathrm{mL})$. However, the QTcF value was lower at $2 \mathrm{~h}$ post dose despite higher drug concentrations [QTcF of $446 \mathrm{~ms}$ (change of $58.3 \mathrm{~ms}$ )], with exposure of apalutamide and $\mathrm{N}$-desmethyl apalutamide of 8.77 and $8.87 \mu / \mathrm{mL}$, respectively, suggesting that the increase of more than $60.4 \mathrm{~ms}$ was not consistent at similar exposure within the same individual. No patients discontinued treatment due to QTc prolongation, and no evidence of development of ventricular arrhythmias was observed that could be attributed to underlying QTc prolongation.

According to Sarapa et al., the magnitude of changes in QTcF interval (as observed in the present study) may be considered a mild to moderate QTc-prolonging effect for an anticancer agent, and these same authors have suggested that a dedicated QTc study for anticancer agents that excludes $\Delta$ QTc of $<20 \mathrm{~ms}$ can be concluded as a negative study [21], consistent with the present data showing no new clinical concerns [4]. Our data are supported by a small and voluntary QTc substudy of the SPARTAN trial [6], which also revealed no patients in the apalutamide arm with a QTcF interval $>480 \mathrm{~ms}$. In the placebo arm of the SPARTAN study, two of six patients had at least one postdose QTcF interval $>450$ and $\leq 480 \mathrm{~ms}$; one of these two patients had a baseline QTcF interval $>450 \mathrm{~ms}$.

De novo $\mathrm{T}$-wave abnormalities were observed in three patients (7\%) and were absent at baseline, and no QTcF prolongation $\geq 480 \mathrm{~ms}$ was observed in these three patients. No evidence for an apalutamide treatment effect was noted on the length of the PR interval in our study. The observed mean increases in QRS duration as observed on C3D1 were minimal $(<2.5 \mathrm{~ms})$ and are considered not clinically meaningful.

Overall, exposures of apalutamide and its extent of accumulation observed in this study are consistent with those previously reported [7]. To explore the relationship between apalutamide concentration at steady state and QTcF, PK (plasma concentration) and PD (change from baseline in QTcF) data were analyzed using a linear mixed-effects model. The analysis revealed an association between plasma concentration of apalutamide and QTcF and predicted a prolongation of $13.81 \mathrm{~ms}$ at $C_{\max }$ at steady state (C3D1), with an upper bound of two-sided 90\% CI of $17.85 \mathrm{~ms}$. Because of the correlation between apalutamide and $N$-desmethyl apalutamide exposures, a similar association between plasma concentration of $N$-desmethyl apalutamide and QTcF was detected. Based on the flat PK profile of $\mathrm{N}$-desmethyl apalutamide at steady state, the apparent association between $N$-desmethyl apalutamide concentration and QTcF at steady state is considered less clinically meaningful compared with the parent drug. Overall, results of the PK/PD analysis indicated that a large effect on $\triangle \mathrm{QTcF}$ is not expected at steady state following $240 \mathrm{mg}$ daily dose of apalutamide.

These data from a Phase Ib QT/QTc study that investigated the effect of apalutamide on QTc intervals revealed no new safety signals associated with apalutamide treatment in men with CRPC. For the primary endpoint, no significant safety findings related to QT prolongation were documented and there were no observed arrhythmias related to apalutamide. Overall, the safety profile observed in this study was as expected based on the known safety profile of apalutamide and results from other studies [6, 7, 19,20].

Overall, these data demonstrate that apalutamide does not produce clinically meaningful changes in QTc interval or produce a concerning effect on ventricular repolarization in patients with CRPC. 
Acknowledgements This study was funded by Janssen Research \& Development. Writing assistance was provided by Brian Haas, $\mathrm{PhD}$, of PAREXEL, and was funded by Janssen Global Services, LLC.

Funding This study was funded by Janssen Research \& Development.

\section{Compliance with ethical standards}

Conflict of interest Bodine Belderbos has held a consulting/advisory role and has received travel/accommodations/expenses from Astellas. Ronald de Wit has received advisory fees from Sanofi, Merck, Roche, and Lilly, and research grants from Erasmus MC Cancer Institute, Sanofi, and Bayer. Caly Chien is employed by Janssen Research \& Development, LLC, and holds stock in Johnson \& Johnson. Anna Mitselos is employed by Janssen Research \& Development, LLC, and holds stock in Johnson \& Johnson. Peter Hellemans is employed by Janssen Research \& Development, LLC, and holds stock in Johnson \& Johnson. James Jiao is employed by Janssen Research \& Development, LLC, and holds stock in Johnson \& Johnson. Margaret K. Yu is employed by Janssen Research \& Development, LLC, and holds stock in Johnson \& Johnson. Gerhardt Attard has received honoraria from Janssen and Astellas; has held a consulting or advisory role at Janssen, Astellas, Pfizer, Millennium Pharmaceuticals, Abbott Laboratories, Essa Pharmaceuticals, Bayer Pharmaceuticals, Veridex, Ventana, and Novartis; has served on speaker's bureau for Janssen, Astellas, Takeda, SanofiAventis, and Ventana; has received research funding from Janssen, AstraZeneca, Arno Therapeutics, and Innocrin Pharma; holds patents, receives royalties, or has intellectual property with Institute of Cancer Research [ICR] rewards to inventors list of abiraterone acetate; has received travel, accommodations, and expenses from Janssen, Astellas, Pfizer, Ventana, Abbott Laboratories, Bayer Pharmaceuticals, and Essa Pharmaceuticals; and was employed by ICR through 8 Jan 2018. Iurie Bulat has nothing to disclose. Jeffrey W. Edenfield has nothing to disclose. Fred Saad has received grants, personal fees, and nonfinancial support from Janssen, Astellas, Sanofi, and Bayer.

Ethical approval All procedures performed in studies involving human participants were in accordance with the ethical standards of the institutional and/or national research committee and with the 1964 Helsinki declaration and its later amendments or comparable ethical standards.

Informed consent Informed consent was obtained from all individual participants included in the study.

Open Access This article is distributed under the terms of the Creative Commons Attribution 4.0 International License (http://creativeco mmons.org/licenses/by/4.0/), which permits unrestricted use, distribution, and reproduction in any medium, provided you give appropriate credit to the original author(s) and the source, provide a link to the Creative Commons license, and indicate if changes were made.

\section{References}

1. Ferlay JSI, Ervik M, Dikshit R, Eser S, Mathers C, Rebelo M, Parkin DM, Forman D, Bray F (2012) GLOBOCAN 2012 v1.0, Cancer Incidence and Mortality Worldwide: IARC CancerBase No. 11. http://globocan.iarc.fr. Accessed 28 Feb 2018

2. Gartrell BA, Saad F (2014) Managing bone metastases and reducing skeletal related events in prostate cancer. Nat Rev Clin Oncol 11(6):335-345. https://doi.org/10.1038/nrclinonc.2014.70
3. Tan MH, Li J, Xu HE, Melcher K, Yong EL (2015) Androgen receptor: structure, role in prostate cancer and drug discovery. Acta Pharmacol Sin 36(1):3-23. https://doi.org/10.1038/ aps. 2014.18

4. ERLEADA [prescribing information] (2018). Available via Janssen website. http://www.janssenlabels.com/package-insert/ product-monograph/prescribing-information/ERLEADA-pi.pdf. Accessed 2 July 2018

5. Clegg NJ, Wongvipat J, Joseph JD, Tran C, Ouk S, Dilhas A, Chen Y, Grillot K, Bischoff ED, Cai L, Aparicio A, Dorow S, Arora V, Shao G, Qian J, Zhao H, Yang G, Cao C, Sensintaffar J, Wasielewska T, Herbert MR, Bonnefous C, Darimont B, Scher HI, Smith-Jones P, Klang M, Smith ND, De Stanchina E, Wu N, Ouerfelli O, Rix PJ, Heyman RA, Jung ME, Sawyers CL, Hager JH (2012) ARN-509: a novel antiandrogen for prostate cancer treatment. Cancer Res 72(6):1494-1503. https://doi. org/10.1158/0008-5472.CAN-11-3948

6. Smith MR, Saad F, Chowdhury S, Oudard S, Hadaschik BA, Graff JN, Olmos D, Mainwaring PN, Lee JY, Uemura H, Lopez-Gitlitz A, Trudel GC, Espina BM, Shu Y, Park YC, Rackoff WR, Yu MK, Small EJ, Investigators S (2018) Apalutamide treatment and metastasis-free survival in prostate cancer. N Engl J Med 378(15):1408-1418. https://doi.org/10.1056/NEJMoa1715546

7. Rathkopf DE, Morris MJ, Fox JJ, Danila DC, Slovin SF, Hager JH, Rix PJ, Chow Maneval E, Chen I, Gonen M, Fleisher M, Larson SM, Sawyers CL, Scher HI (2013) Phase I study of ARN509, a novel antiandrogen, in the treatment of castration-resistant prostate cancer. J Clin Oncol 31(28):3525-3530. https://doi. org/10.1200/JCO.2013.50.1684

8. Levine GN, D'Amico AV, Berger P, Clark PE, Eckel RH, Keating NL, Milani RV, Sagalowsky AI, Smith MR, Zakai N (2010) Androgen-deprivation therapy in prostate cancer and cardiovascular risk: a science advisory from the American Heart Association, American Cancer Society, and American Urological Association: endorsed by the American Society for Radiation Oncology. CA Cancer J Clin 60(3):194-201. https://doi.org/10.3322/caac.20061

9. Van Poppel H, Tombal B (2011) Cardiovascular risk during hormonal treatment in patients with prostate cancer. Cancer Manag Res 3:49-55. https://doi.org/10.2147/CMR.S16893

10. CASODEX [prescribing information] (2017). Available via AZ PI Central website. https://www.azpicentral.com/casodex/casod ex.pdf. Accessed 2 July 2018

11. Garnick MB, Pratt CM, Campion M, Shipley J (2004) The effect of hormonal therapy for prostate cancer on the electrocardiographic QT interval: phase 3 results following treatment with leuprolide and goserelin, alone or with bicalutamide, and the $\mathrm{GnRH}$ antagonist abarelix. J Clin Oncol 22(14_suppl):4578. https://doi. org/10.1200/jco.2004.22.14_suppl.4578

12. CASODEX summary of product characteristics (2018). Available via emc+ website. https://www.medicines.org.uk/emc/produ ct/3805/smpc. Accessed 2 July 2018

13. Scher HI, Fizazi K, Saad F, Taplin ME, Sternberg CN, Miller K, de Wit R, Mulders P, Chi KN, Shore ND, Armstrong AJ, Flaig TW, Flechon A, Mainwaring P, Fleming M, Hainsworth JD, Hirmand M, Selby B, Seely L, de Bono JS, Investigators A (2012) Increased survival with enzalutamide in prostate cancer after chemotherapy. N Engl J Med 367(13):1187-1197. https://doi. org/10.1056/NEJMoa1207506

14. Siemens DR, Klotz L, Heidenreich A, Chowdhury S, Villers A, Baron B, van Os S, Hasabou N, Wang F, Lin P, Shore ND (2018) Efficacy and safety of enzalutamide vs bicalutamide in younger and older patients with metastatic castration resistant prostate cancer in the TERRAIN trial. J Urol 199(1):147-154. https://doi. org/10.1016/j.juro.2017.08.080

15. Camm AJ (2005) Clinical trial design to evaluate the effects of drugs on cardiac repolarization: current state of the art. Heart 
Rhythm 2(2 Suppl):S23-S29. https://doi.org/10.1016/j.hrthm 2004.09.019

16. Morganroth J (2004) A definitive or thorough phase 1 QT ECG trial as a requirement for drug safety assessment. J Electrocardiol 37(1):25-29

17. U.S. Department of Health and Human Services, Food and Drug Administration, Center for Drug Evaluation and Research (CDER), Center for Biologics Evaluation and Research (CBER) (2012) E14 clinical evaluation of QT/QTc interval prolongation and proarrhythmic potential for non-antiarrhythmic drugs-questions and answers (R1). October $2012 \mathrm{ICH}$. Available via the US Food and Drug Administration website. https://www.fda.gov/ drugs/guidancecomplianceregulatoryinformation/guidances/ ucm323656.htm. Accessed 3 July 2018

18. Fridericia LS (2003) The duration of systole in an electrocardiogram in normal humans and in patients with heart disease. 1920. Ann Noninvasive Electrocardiol 8(4):343-351

19. Rathkopf DE, Antonarakis ES, Shore ND, Tutrone RF, Alumkal JJ, Ryan CJ, Saleh M, Hauke RJ, Bandekar R, Maneval EC, de
Boer CJ, Yu MK, Scher HI (2017) Safety and Antitumor Activity of Apalutamide (ARN-509) in metastatic castration-resistant prostate cancer with and without prior abiraterone acetate and prednisone. Clin Cancer Res 23(14):3544-3551. https://doi. org/10.1158/1078-0432.CCR-16-2509

20. Smith MR, Antonarakis ES, Ryan CJ, Berry WR, Shore ND, Liu G, Alumkal JJ, Higano CS, Chow Maneval E, Bandekar R, de Boer CJ, Yu MK, Rathkopf DE (2016) Phase 2 study of the safety and antitumor activity of apalutamide (ARN-509), a potent androgen receptor antagonist, in the high-risk nonmetastatic castrationresistant prostate cancer cohort. Eur Urol 70(6):963-970. https:// doi.org/10.1016/j.eururo.2016.04.023

21. Sarapa N, Britto MR (2008) Challenges of characterizing proarrhythmic risk due to QTc prolongation induced by nonadjuvant anticancer agents. Expert Opin Drug Saf 7(3):305-318. https:// doi.org/10.1517/14740338.7.3.305 